\title{
NUMERICAL INTEGRATION OF VECTOR FIELDS OVER CURVES WITH ZERO AREA
}

\author{
JENNY C. HARRISON
}

(Communicated by J. Marshall Ash)

\begin{abstract}
The boundary of a Jordan domain $A$ may be a nonsmooth curve $\gamma$. If $F$ is a smooth vector field defined near $\gamma$, then $F$ is integrable over $\gamma$ provided $\gamma$ has two-dimensional Lebesgue measure zero and $F$ is sufficiently smooth. When actually computing the integral $\int_{\gamma} F \cdot d s$, one might hope that $\lim _{k \rightarrow \infty} \int_{\gamma_{k}} F \cdot d s=\int_{\gamma} F \cdot d s$ for PL or smooth approximators $\gamma_{k}$ of $\gamma$. Several examples show that this is not the case. However, there are algorithms for choosing $\gamma_{k}$ so that $\int_{\gamma_{k}} F \cdot d s$ converges to $\int_{\gamma} F \cdot d s$ exponentially quickly.
\end{abstract}

\section{INTRODUCTION AND PRELIMINARIES}

Let $S^{1}$ denote the unit circle, oriented positively. A Jordan curve $\gamma$ is the image of a 1-1, continuous mapping $\varphi: S^{1} \rightarrow \mathbb{R}^{2}$. The mapping $\varphi$ is called a parametrization of $\gamma$ and induces an orientation on $\gamma$. The bounded region $D$ which $\gamma$ bounds is called its Jordan domain. Let $m$ denote the twodimensional Lebesgue measure.

Let $F: U \subset \mathbb{R}^{2} \rightarrow \mathbb{R}^{2}$ be a vector field defined on an open subset $U$ of $\mathbb{R}^{2}$ and $\gamma$ a Jordan curve contained in $U$. In case there is a smooth parametrization $\varphi$ of $\gamma$, the line integral $\int_{\gamma} F \cdot d s$ is classically defined by $\int_{0}^{1} F(\varphi(t)) \cdot \varphi^{\prime}(t) d t$.

The answer is independent of $\varphi$. This definition does not make sense if $\gamma$ is without tangent vectors. The line integral has recently been extended to arbitrary continuous Jordan curves with two-dimensional Lebesgue measure zero, provided $F$ is sufficiently smooth. (see [H-N].)

Definitions. Let $K$ be a bounded set in $\mathbb{R}^{2}$. Let $N(\varepsilon)$ denote the minimum number of squares of side length epsilon required to cover $K$. The box dimension of $K$, denoted $\operatorname{dim}(K)$, is defined by

$$
\operatorname{dim}(K)=\lim _{\varepsilon \rightarrow 0}-\frac{\log N(\varepsilon)}{\log \varepsilon},
$$

if the limit exists.

Received by the editors March 26, 1991 and, in revised form, September 28, 1992.

1991 Mathematics Subject Classification. Primary 49Q15; Secondary 65D30.

The author was partially supported by the National Science Foundation VPW Program and the Mathematical Sciences Research Institute. 
A $k$-square from the binary grid is a square of the form

$$
Q=\left[j 2^{-k},(j+1) 2^{-k}\right] \times\left[l 2^{-k},(l+1) 2^{-k}\right]
$$

where $j, l \in \mathbb{Z}$. One need only consider $k$-squares to calculate $\operatorname{dim}(K)$.

$$
\operatorname{dim}(K)=\lim _{k \rightarrow \infty}-\frac{\log N(k)}{k \log 2},
$$

where $N(k)$ is the minimum number of $k$-squares from the binary grid required to cover $K$.

Let $U$ be an open subset of $\mathbb{R}^{2}$. A continuous function $f: U \rightarrow \mathbb{R}$ is said to be of class $C^{0}$. A function $f: U \rightarrow \mathbb{R}$ is said to be of class $C^{\alpha}, 0<\alpha<1$, if there exists a constant $M>0$ such that $|f(x)-f(y)|<M|x-y|^{\alpha}$ for all $x, y \in U$. Define $\mathbb{Z}^{+}$to be the set of integers $\geq 1$. We say $f$ is of class $C^{s}, s=r+\alpha, r \in \mathbb{Z}^{+}, 0 \leq \alpha<1$, if the $r$ th partial derivatives of $f$ exist and are of class $C^{\alpha}$. If $f$ is defined on a closed subset $K$ of $\mathbb{R}^{2}$, it is said to be of class $C^{s}$ if it extends to a function of class $C^{S}$ defined in an open neighborhood $U$ of $K$. A function is smooth if it is of class $C^{1}$. A vector field $F: U \subset \mathbb{R}^{2} \rightarrow \mathbb{R}^{2}$ is of class $C^{S}$ if each of its component functions $F_{1}$ and $F_{2}$ is of class $C^{S}$. For $p \in U$, define $|F(p)|=\sqrt{F_{1}(p)^{2}+F_{2}(p)^{2}}$. Define $|F|_{0}=\sup \{|F(p)|: p \in U\}$. If $F \in C^{\alpha}, 0<\alpha<1$, the $\alpha$-norm of $F$ is defined by

$$
|F|_{\alpha}=|F|_{0}+\sup \left\{\frac{|F(p)-F(q)|}{|p-q|^{\alpha}}, p, q \in U, p \neq q\right\} .
$$

For curves with two-dimensional Lebesgue measure zero, the existence of $\int_{\gamma} F \cdot d s$ is a consequence of the assumption that $F$ has Hölder exponent larger than the box dimension of $\gamma$ minus 1 . (See [H-N].)

An arc $\sigma$ is the image of a 1-1 continuous mapping $\varphi:[0,1] \rightarrow \mathbb{R}$, oriented so that $\sigma(0)<\sigma(1)$. We will show that the theory of integration over Jordan curves does not extend to all arcs in the plane, but it is valid for arcs with finitely accessible endpoints: $\sigma$ has finitely accessible endpoints if there exists a rectifiable arc $\tau$ so that $\sigma \cup \tau$ is a Jordan curve and $\sigma \cap \tau=\{\sigma(0), \sigma(1)\}$.

For $1<d \leq 2$, let $\Lambda_{d}=\{(\gamma, F): \gamma$ is a Jordan curve or an arc with finitely accessible endpoints, $\operatorname{dim}(\gamma)<d, m(\gamma)=0$ and $F$ is a vector field defined in a neighborhood of $\gamma$ of class $\left.C^{d-1}\right\}$. It is proved in [H-N] that the line integral extends to elements of $\Lambda_{d}$. That is, there is a real valued function $\int$ defined on $\Lambda_{d}$ such that $\int(\gamma, F)=\int_{\gamma} F \cdot d s$ as long as there is a smooth parametrization of $\gamma$. In general, we denote $\int(\gamma, F)=\int_{\gamma} F \cdot d s$ for $(\gamma, F) \in \Lambda_{d}$. Moreover, the following is proved in [H-N]:

Theorem 1. The mapping $\int: \Lambda_{d} \rightarrow \mathbb{R}$ has the following properties:

(i) Bilinearity:

$$
\begin{gathered}
\int_{\gamma}(a F+b G) \cdot d s=a \int_{\gamma} F \cdot d s+b \int_{\gamma} G \cdot d s \quad \text { for }(\gamma, F),(\gamma, G) \in \Lambda_{d} ; \\
\int_{\gamma_{1}} F \cdot d s+\int_{\gamma_{2}} F \cdot d s=\int_{\gamma_{1} \cup \gamma_{2}} F \cdot d s \quad \text { for }\left(\gamma_{1}, F\right),\left(\gamma_{2}, F\right) \in \Lambda_{d},
\end{gathered}
$$

and $\gamma_{1} \cap \gamma_{2}$ with one-dimensional Lebesgue measure zero. 
(ii) Independence of the extension: If $(\gamma, F),(\gamma, G) \in \Lambda_{d}$ and $\left.F\right|_{\gamma}=\left.G\right|_{\gamma}$ then $\int_{\gamma} F \cdot d s=\int_{\gamma} G \cdot d s$.

(iii) Fundamental theorem of calculus: $\int_{\sigma} \nabla f \cdot d s=f(\sigma(1))-f(\sigma(0))$ for an arc $\sigma,(\sigma, \nabla f) \in \Lambda_{d}$.

(iv) Green's theorem: Suppose $F$ is smooth away from $\gamma, D$ is its Jordan domain and $(\gamma, F) \in \Lambda_{d}$. Then $\int_{\gamma} F \cdot d s=\int_{D}(\nabla \times F) \cdot d A$.

Remarks.

- One may not replace "box dimension" with "Hausdorff dimension". That is, there is no generalized integral defined for all pairs $(\gamma, F)$ with the Hölder exponent of $F$ exceeding the Hausdorff dimension of $\gamma$ minus one that satisfies both (ii) and (iv). (see [H1].)

- Theorem 1 does not extend to all arcs. Let $\sigma$ be the arc depicted in Figure 1 and $F$ the analytic vector field $F(x, y)=(y, 0)$. Let $\sigma_{k}$ denote the portion of $\sigma$ outside the $1 / k$-neighborhood of the origin so that $\sigma_{k} \rightarrow \sigma$, pointwise. Clearly, if there are enough loops in the spiral the integral of $F$ along $\sigma_{k}$ will diverge as $k \rightarrow \infty$.

Note that $\sigma$ does not have finitely accessible endpoints and therefore does not contradict Theorem 1.

- If $m(\gamma)>0$ then (iv) cannot be satisfied. Let $D$ be the Jordan domain of $\gamma$. Assume that $\gamma$ is a subset of the unit disk $A$. Let $A^{\prime}=A \backslash D$ and $F(x, y)=(0, x)$. If the integral $\int_{\gamma} x d y$ exists and satisfies (iv) then it equals $\int_{D} d x d y$. Applying (iv) again gives

$$
\int_{\gamma} x d y=\int_{S^{1}} x d y-\int_{A^{\prime}} d x d y=\int_{A} d x d y-\int_{A^{\prime}} d x d y .
$$

Hence $m(A)=m(D)+m\left(A^{\prime}\right)$, contradicting the assumption that $m(\gamma)>0$.

- If $\operatorname{dim}(\gamma)=1+\alpha$ the result fails. Hassler Whitney constructed a function "nonconstant on a connected set $\gamma$ of critical points". Properties (ii) and (iii) of Theorem 1 cannot both be satisfied. Otherwise, by (iii), $\int_{\sigma} \nabla f \cdot d s=f(\sigma(b))-$ $f(\sigma(a))$. The left-hand side of the equation is zero by (ii) applied to $G \equiv 0$. The right-hand side is nonzero since $f$ is nonconstant on $\sigma$. It is not hard to show that $\nabla f \in C^{\alpha}$ and $\operatorname{dim}(\gamma)=1+\alpha$ so Theorem 1 is "sharp". (See [W, N, H-N].)

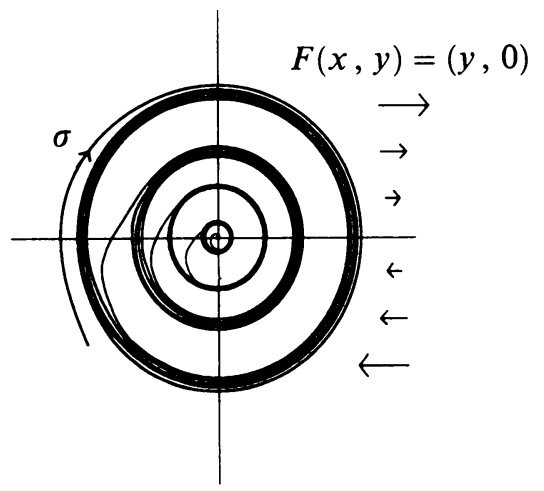

Figure 1. An arc $\sigma$ for which the line integral is not defined. 


\section{TWO COUNTEREXAMPLES}

Since tangent vectors do not generally exist for continuous Jordan curves, conventional methods of integration are not valid. However, the theory guarantees the existence of $\int_{\gamma} F \cdot d s$, provided $(\gamma, F) \in \Lambda_{d}$. If $F$ is of class $C^{1}$ and $m(\gamma)=0$, one may always calculate $\int_{A}(\nabla \times F) \cdot d A$ and this will equal $\int_{\gamma} F \cdot d s$.

If $F$ is only of class $C^{\alpha}$, the integral is defined using the Whitney extension theorem: $F$ may be defined on all of $\mathbb{R}^{2}$. Restrict $F$ to $\gamma$, then extend $\left.F\right|_{\gamma}$ to a new vector field $G$ on $\mathbb{R}^{2}$ that is $C^{\infty}$ away from $\gamma$ and globally of class $C^{\alpha}$. The old values of $F$ away from $\gamma$ are completely discarded. Now one may use the curl formulation and define

$$
\int_{\gamma} F \cdot d s=\int_{A}(\nabla \times G) \cdot d A .
$$

This integral exists and is independent of the extension $G$. (See [H-N].) However, it is not practical to use the Whitney extension theorem in applications. A natural approach to actually finding the integral would be to integrate over approximating curves that are smooth or PL. There is no hope that the limit will generally converge to anything meaningful if the hypotheses in Theorem 1 are not met, so we henceforth assume that $(\gamma, F) \in \Lambda_{d}$. That is, $m(\gamma)=0$, $\operatorname{dim}(\gamma)<d$ and $F \in C^{d-1}$.

Even if the hypotheses are met, there can be problems as the next examples show.

Let $\gamma$ be a Jordan curve. We say $\left\{\gamma_{k}\right\}$ approximates $\gamma$ if $\gamma_{k}$ converges to $\gamma$ in the Hausdorff metric and $\gamma_{k}$ is oriented in the same way as $\gamma$. We write $\gamma_{k} \rightarrow \gamma$. There are two natural classes of approximators of $\gamma$ :

(a) $S$ is a secant approximator of $\gamma$ if $S$ is PL and its vertices are contained in $\gamma$ and have the same cyclical order in $S$ as in $\gamma$. That is, suppose $x_{i} \in \gamma$, $0 \leq i \leq n$, with $x_{0}<x_{1}<\cdots<x_{n}=x_{0}$ in $\gamma$. Then $X=\left\{x_{i}, 0 \leq i \leq\right.$ $n\}$ forms an ordered partition of $\gamma$. An ordered partition $X$ determines an oriented PL curve $S_{X}$ called a secant approximator of $\gamma$ which is the union of the oriented straight line segments in $\mathbb{R}^{2}$ with endpoints $x_{i}<x_{i+1}$.

(b) $S$ is an internal approximator of $\gamma$ if $S$ is contained in the Jordan domain bounded by $\gamma$. (Of course, one may equally consider external approximators.) We require that $S$ be either smooth or PL.

We next show even if the integral $\int_{\gamma} F \cdot d s$ exists, not all secant or internal approximators of $\gamma$ may be used to approximate the integral. That is, there exist $\gamma_{k} \rightarrow \gamma$ with $\int_{\gamma_{k}} F \cdot d s \nrightarrow \int_{\gamma} F \cdot d s$.

Example 1. The integral of an analytic vector field $F$ over a secant approximator of a Jordan curve $\gamma$ does not necessarily approximate the integral of $F$ over $\gamma$.

Consider the vector field $F(x, y)=(y, 0)$ and the Jordan curve $\gamma$ illustrated in Figure 2. The curve $\gamma$ is the countable union of arcs $\sigma_{i}$. Each arc $\sigma_{i}$ contains many "thin" half-moon turns along which the integral of $F$ is virtually zero so that $\int_{\gamma} F \cdot d s>0$. The secant approximator $\gamma_{k}$ closely approximates $\sigma_{1}, \ldots, \sigma_{k}$ so that the integrals of $F$ over $U \sigma_{i}, i=1, \ldots, k$, and $\gamma_{k}$ are 


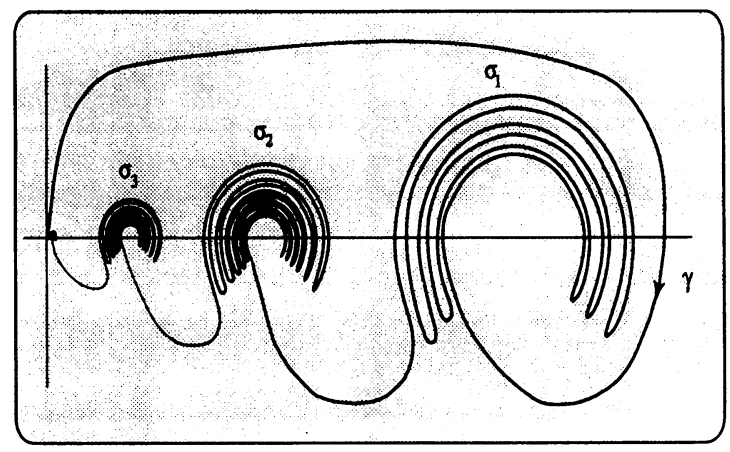

(a)
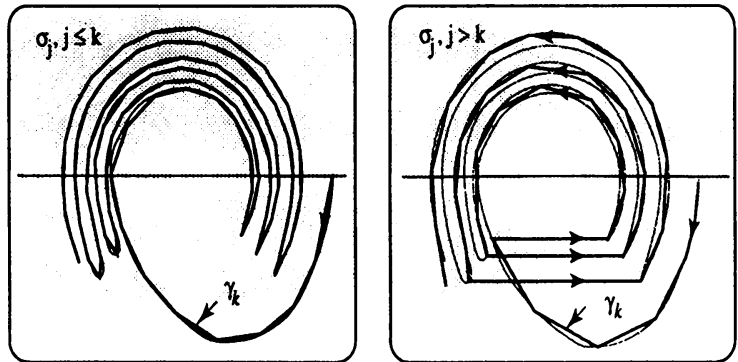

(b)

Figure 2. (a) The curve $\gamma$ is a countable union of arcs $\sigma_{l}$. (b) The secant approximator $\gamma_{k}$ on each subarc of $\gamma$.

essentially the same. The figure indicates how $\gamma_{k}$ is defined over the arcs $\sigma_{i}$, $k<i \leq k^{2}$. It is not hard to see that with a little care, $\int_{\sigma_{k}} F \cdot d s<0$.

This is a counterexample to a conjecture of $P$. Jones that $\int_{\gamma_{k}} F \cdot d s \rightarrow \int_{\gamma} F \cdot d s$ if $\gamma_{k}$ is a secant approximator of $\gamma$ with $\gamma_{k} \rightarrow \gamma$ and the pair $(\gamma, F) \in \Lambda_{d}$. In the next section we provide an algorithm for finding secant approximators over which the integral do converge to the correct limit $\int_{\gamma} F \cdot d s$.

Example 2. The integral of a vector field $F$ over an internal approximator of a Jordan curve $\gamma$ does not approximate the integral of $F$ over $\gamma$.

Let $\gamma$ denote the boundary of the unit square, $[0,1] \times[0,1]$ and $\gamma_{k}$ the internal approximator depicted in Figure 3 on the next page. Let

$$
f(x)= \begin{cases}0 & \text { if } x<0 \text { or } x>1, \\ (-1)^{n+1} / n & \text { for } x=1 / n \text { and } n=2,3, \ldots\end{cases}
$$

Extend $f$ to the rest of of $\mathbb{R}$ so that $f$ has Hölder exponent $1 / 2$. 


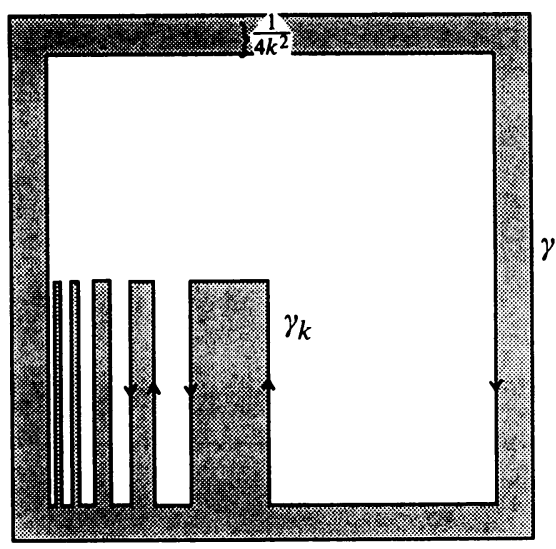

FIGURE 3

Let $F$ be the vector field $F(x, y)=(0, f(x))$. By Theorem 1, the integral of $F$ over $\gamma$ exists. It is 0 by (ii). For $n \geq 1$, let

$$
I_{n}=\left\{\frac{1}{n}\right\} \times\left[\frac{1}{n}, 1-\frac{1}{n}\right]
$$

and

$$
J_{n}= \begin{cases}{\left[\frac{1}{n+1}, \frac{1}{n}\right] \times\left\{\frac{1}{n}\right\}} & \text { if } n \text { is odd, } \\ {\left[\frac{1}{n+1}, \frac{1}{n}\right] \times\left\{1-\frac{1}{n}\right\}} & \text { if } n \text { is even. }\end{cases}
$$

Define $\gamma_{k}=\bigcup I_{n} \cup J_{n}, 2 k \leq n \leq(2 k)^{2}-1$, together with the five segments of the $\left(1-1 / 4 k^{2}\right)$-square which make $\gamma_{k}$ into a Jordan curve. (See Figure 3.) Note $\int_{I_{n} \cup J_{n}} F \cdot d s=(1 / n)(1-2 / n)$. It follows easily that $\lim \int_{\gamma_{k}} F \cdot d s$ grows as $\log k$.

A version of this example may be found in [H-N].

\section{Two ALGorithmS}

We describe algorithms for choosing secant approximators and internal approximators that can be used to estimate the integral of $F$ over $\gamma$.

Definitions. If $U \subset \mathbb{R}^{2}$ is nonempty, the diameter of $U$ is defined by $|U|=$ $\sup \{|x-y|: x, y \in U\}$. Let $\lambda=\left\{\lambda_{i}\right\}$ be a collection of subsets of $\mathbb{R}^{2}$ and $d>0$. The number $\sum_{i}\left|\lambda_{i}\right|^{d}$ is called the $d$-sum of $\lambda$. If $A=\bigcup T_{i}$ and the $T_{i}$ are closed domains with disjoint interiors, we call $T=\left\{T_{i}\right\}$ a decomposition of $A$.

Let $K \subset \mathbb{R}^{2}$ be closed. We decompose $\mathbb{R}^{2} \backslash K$ into $k$-squares $Q$ of the form $Q=\left[j 2^{-k},(j+1) 2^{-k}\right] \times\left[l 2^{-k},(l+1) 2^{-k}\right]$ where $j, l \in \mathbb{Z}:$ Fix $k \in \mathbb{Z}$ and consider the collection of $k$-squares in $\mathbb{R}^{2}$ that do not touch $K$ or a $k$ square that touches $K$. Take the union over $k$, discarding any squares that are contained in larger ones. The final collection $W$ is a decomposition of $\mathbb{R}^{2} \backslash K$ and is called the Whitney decomposition of $\mathbb{R}^{2} \backslash K$ (see Figure 4). It satisfies $|Q| \leq d(Q, K) \leq 4|Q|, Q \in \mathbf{W}$. Define $A_{k}$ to be the union of all $j$-squares of $\mathbf{W}, j \leq k$. 


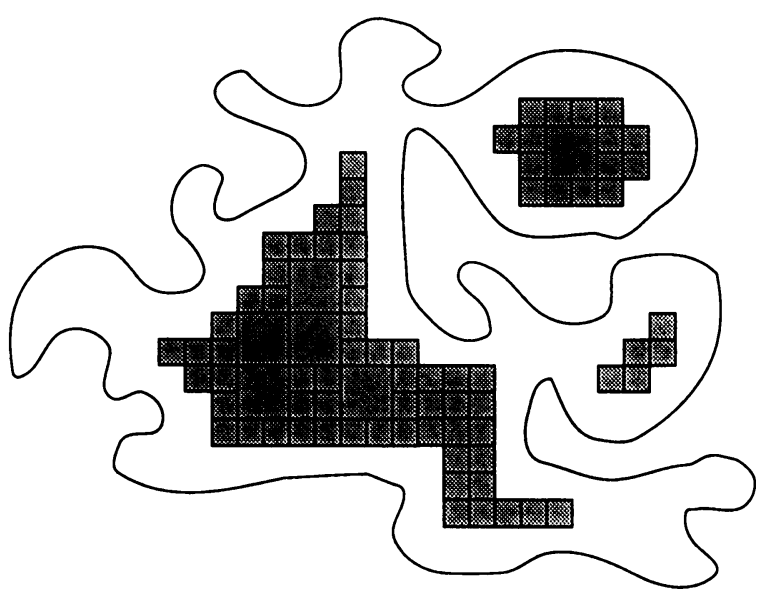

FIGURE 4. The Whitney decomposition of the Jordan domain of $\gamma$.

Theorem 2 (Continuity). Let $d>1$. There exists $C$ such that if $\left(\gamma_{1}, F\right)$, $\left(\gamma_{2}, F\right) \in \Lambda_{d}$ and $\gamma_{1}$ and $\gamma_{2}$ bound a region $A$ decomposed by $\lambda$ then

$$
\left|\int_{\gamma_{1}} F \cdot d s-\int_{\gamma_{2}} F \cdot d s\right| \leq C|F|_{d-1} \times d-\operatorname{sum}(\lambda) .
$$

For the proof, see [H-N, Theorem 3.7].

This theorem gives us an idea of which curves are good approximators. The region bounded by $\gamma_{k}$ and $\gamma$ should have a decomposition with small $d$-sum. We make an explicit choice.

Definition. Let $\gamma$ be a Jordan curve and $C \geq 1$. A sequence $\left\{\gamma_{k}\right\}$ of secant approximators of $\gamma$ is said to be $C$-regular if there exists $N$ such that if $k \geq N$ then (i) $\gamma_{k}$ is a Jordan curve oriented in the same direction as $\gamma$; (ii) any pair of successive vertices of $\gamma_{k}$ is contained in the boundary of a $k$-square meeting $\gamma$; (iii) there are $\leq C$ such pairs in any single $k$-square meeting $\gamma$.

A sequence $\left\{\sigma_{k}\right\}$ of secant approximators of an arc $\sigma$ is said to be $C$ regular if $\sigma_{k}$ contains $\sigma(0)$ and $\sigma(1)$ and (i) $\sigma_{k}$ is embedded; (ii) any pair of successive vertices of $\sigma_{k}$ are contained in the boundary of a $k$-square meeting $\sigma$, possibly excepting the endpoints; (iii) there are $\leq C$ such pairs in any $k$ square meeting $\sigma$.

Algorithm for choosing secant approximators. Let $\sigma$ be an arc. There exists a 1-regular sequence $\left\{\sigma_{k}\right\}$ of secant approximators of $\sigma$. If $\gamma$ is a Jordan curve, there exists a 3-regular sequence of secant approximators of $\gamma$.

Construction. Let $p$ and $q$ be the endpoints of $\sigma$. Fix $k \geq 1$. Let $T_{1}$ be the union of $k$-squares that contain $x_{0}=p$. If $\sigma$ exists at all from $T_{1}$, let $x_{1}$ be the last exit point. (If $\sigma$ does not exit $T_{1}$, let $x_{1}=q$ and stop the process.) Let $T_{2}$ be the union of those $k$-squares not in $T_{1}$ that contain $x_{1}$. Let $x_{2}$ be the last exit point of $\sigma$ from $T_{2}$, if it exists $T_{2}$ at all. (If $\sigma$ does not exist $T_{2}$, let $x_{2}=q$.) Inductively define $x_{i}$, forming a partition $X_{k}=\left\{x_{i}\right\}$, stopping when $q$ is reached. Then $\sigma_{k}=S_{X_{k}}$ contains the endpoints $p$ and $q$. The $T_{i}$ 
have disjoint interiors. Since $\sigma_{k}$ contains at most one segment in each $T_{i}$, it is embedded. (The only possible ambiguity is at the vertices $x_{i}$, but since $\sigma$ is embedded and $x_{i} \in \sigma$, there is no intersection at the vertices.) A pair of successive vertices of $\sigma_{k}$ lies on the boundary of some $k$-square. Since there is only one such pair, at most, $\left\{\sigma_{k}\right\}$ is 1-regular.

While constructing secant approximators to Jordan curves we prove a slightly stronger statement that will be useful later:

(*) If $\left\{\sigma_{k}\right\}$ is a $C$-regular sequence of secant approximators of $\sigma$ and $\tau$ is any connecting arc for $\sigma$, there exist arcs $\tau_{k} \rightarrow \tau$ so that $\left\{\tau_{k}\right\}$ is a $(C+1)$-regular sequence of secant approximators to $\tau$ and $\left\{\sigma_{k} \cup \tau_{k}\right\}$ is a $(2 C+1)$-regular sequence of secant approximators of $\gamma$.

Let $\left\{\sigma_{k}\right\}$ be a regular sequence of secant approximators converging to $\sigma$ and $\tau$ a connecting arc for $\sigma$. We construct a partition $Y_{k}$ of $\tau$ so that $\tau_{k}=S_{Y_{k}}$ meets $\sigma_{k}$ only at $p$ and $q$, and $\left\{\tau_{k}\right\}$ is a regular sequence of secant approximators of $\tau$. The method is similar to that preceding. Replace the $k$-squares with the components of $T \backslash \sigma_{k}$ for $T$ a $k$-square. By assumption, there are at most $C+1$ of these components $U$. Let $U_{1}$ denote the union of those components with $q$ in their closure and set $y_{0}=q$. Let $y_{1}$ be the last exit point of $\tau$ from $U_{1}$. Observe that the line segment with endpoints $y_{0}$ and $y_{1}$ misses $\sigma_{k}$ except at $q$. Begin the process again with $y_{1}$ and obtain a partition $Y=\left\{q=y_{0}, y_{1}, \ldots, y_{n}=p\right\}$. The secant approximators $\tau_{k}=S_{Y}$ form a $(C+1)$-regular sequence. Hence $\left\{\sigma_{k} \cup \tau_{k}\right\}$ is $(2 C+1)$-regular.

Theorem 3. Suppose $(\lambda, F) \in \Lambda_{d}$. If $\left\{\lambda_{k}\right\}$ is a C-regular sequence of secant approximators of $\lambda$ then

$$
\int_{\lambda} F \cdot d s=\lim _{k \rightarrow \infty} \int_{\lambda_{k}} F \cdot d s .
$$

Proof. Suppose that $\lambda=\gamma$ is a Jordan curve. By the Continuity Theorem we merely have to show that the region $Y_{k}$ bounded by $\gamma$ and $\gamma_{k}$ has a decomposition with small $d$-sum.

Recall the sets $A_{n}$ determined by the Whitney decomposition $\mathbf{W}$ of $\mathbb{R}^{2} \backslash \gamma$ :

$$
A_{n}=\bigcup Q: Q \text { is a } j \text {-square of } \mathbf{w}, \quad j \leq n .
$$

The region $Y_{k}$ is contained in $\mathbb{R}^{2} \backslash A_{n_{k}}$ with $n_{k}$ sufficiently large. Each $Y_{k}$ is the union of those $Q \in \mathbf{W}$ that are completely contained in $Y_{k}$ and the closure of the components of $Q \backslash \gamma_{k}$ that are contained in $Y_{k}$. This induces a decomposition $\mathscr{T}_{k}$ of $Y_{k}$. The curve $\gamma_{k}$ only intersects $k$-squares meeting $\gamma$ and does so at most $C$ times. Thus it meets each $Q \in \mathbf{W}$ at most $C$ times. It follows that the $d$-sum of $\mathscr{T}_{k}$ is bounded $(C+1) \times$ the $d$-sum of $\mathbf{W}$ restricted to $\mathbb{R}^{2} \backslash A_{n_{k}}$. The latter sum $\rightarrow 0$ as $k \rightarrow \infty$; therefore the former sum $\rightarrow 0$.

For arcs $\lambda=\sigma$, first connect the endpoints of $\sigma$ with a rectifiable arc $\tau$. Assume that $\tau$ is smooth away from its endpoints. Apply $(*)$ to find $\tau_{k}$ so that $\left\{\sigma_{k} \cup \tau_{k}\right\}$ is $(2 C+1)$-regular sequence of secant approximators of $\sigma \cup \tau$. Since $\sigma \cup \tau$ is a Jordan curve we may apply the first part to conclude that $\int_{\sigma_{k} \cup \tau_{k}} F \cdot d s \rightarrow \int_{\sigma \cup \tau} F \cdot d s$. By Theorem $1(\mathrm{i})$ we have $\int_{\sigma_{k}} F \cdot d s+\int_{\tau_{k}} F \cdot d s \rightarrow$ $\int_{\sigma} F \cdot d s+\int_{\tau} F \cdot d s$. Since $\tau$ is smooth away from its endpoints standard methods show that $\int_{\tau_{k}} F \cdot d s \rightarrow \int_{\tau} F \cdot d s$. Therefore $\int_{\sigma_{k}} F \cdot d s \rightarrow \int_{\sigma} F \cdot d s$. 
Speed of convergence. Let $d>1$ and $(\gamma, F) \in \Lambda_{d}$. For any $0<\varepsilon<d-\operatorname{dim}(\gamma)$ there exists $C>0$ such that

$$
\left|\int_{\gamma_{k}} F \cdot d s-\int_{\gamma} F \cdot d s\right| \leq C|F|_{d-1} 2^{-k(d-\operatorname{dim}(\gamma)-\varepsilon)} .
$$

To see this, let $Y_{k}$ be the region bounded by $\gamma$ and $\gamma_{k}$. Recall the decomposition $T_{k}$ of $Y_{k}$ induced by the Whitney decomposition of $\mathbb{R}^{2} \backslash \gamma$ (see the proof of Theorem 3). By Theorem 2

$$
\left|\int_{\gamma_{k}} F \cdot d s-\int_{\gamma} F \cdot d s\right| \leq C_{1}|F|_{d-1} \times d-\operatorname{sum}\left(T_{k}\right) .
$$

It follows easily from the definition of box dimension that

(**) The number of $k$-squares in the Whitney decomposition of $\mathbb{R}^{2} \backslash \gamma$ is bounded by $2^{k(\operatorname{dim} \gamma+\varepsilon k)}$, where $\varepsilon_{k} \rightarrow 0$.

Thus

$$
d-\operatorname{sum}\left(T_{k}\right) \leq \sum_{n=k}^{\infty} 2^{\left(\operatorname{dim} \gamma+\varepsilon_{n}-d\right) n} \leq C_{2} \sum_{n=k}^{\infty} 2^{(\operatorname{dim} \gamma+\varepsilon-d) n} .
$$

Since $\varepsilon<d-\operatorname{dim}(\gamma)$, there exists $C_{3}$ such that $2^{\operatorname{dim} \gamma+\varepsilon-d}<1-1 / C_{3}$. Therefore, $d$-sum $\left(T_{k}\right) \leq C_{2} C_{3} 2^{-k(d-\operatorname{dim}(\gamma)-\varepsilon)}$.

Algorithm for choosing internal approximators. Recall $A_{k}$ determined by the Whitney decomposition $\mathbf{W}$ of $\mathbb{R}^{2} \backslash \gamma$. Define $\gamma_{k}=\partial A_{k}$. For $k$ sufficiently large, $\gamma_{k}$ is nonempty. It is a union of segments of length $2^{-k}$, each from the boundary of a $k$-square of $\mathbf{W}$. One may use $\gamma_{k}$ to approximate the integral (see [H-N]) or, more simply, consider the sum of the integrals around each $k$-square that is contained in $\mathbb{R}^{2} \backslash \gamma$ and does not touch any $k$-square which touches $\gamma$. It is easy to show, using $(* *)$, that the integral along the approximating curves converge exponentially quickly to the limit integral along $\gamma$.

\section{REFERENCES}

[H-N] J. Harrison and A. Norton, Geometric integration on fractal curves in the plane, Indiana J. Math. 40 (1991), 567-594.

[H1] J. Harrison, Box dimension vs. Hausdorff dimension in the theory of geometric integration (in preparation).

[N] A. Norton, Functions not constant on fractal quasi-arcs of critical points, Proc. Amer. Math. Soc. 106 (1989), 397-405.

[W] H. Whitney, A function not constant on a connected set of critical points, Duke Math. J. 1 (1935), 514-517.

Department of Mathematics, University of California, Berkeley, California 94720

E-mail address: harrison@math. berkeley.edu 